\title{
Systematic study of multipactor suppression techniques for a superconducting rf gun
}

\author{
Eden Tafa Tulu* \\ University of Rostock, Albert-Einstein-Str. 2, D-18059 Rostock, Germany \\ Ursula van Rienen \\ University of Rostock, Albert-Einstein-Str. 2, 18059 Rostock, Germany \\ André Arnold \\ Helmholtz-Zentrum Dresden-Rossendorf, Bautzner Landstraße 400, 01328 Dresden, Germany
}

(Received 14 June 2018; published 30 November 2018)

\begin{abstract}
Future light sources such as synchrotron radiation sources driven by an energy recovery linac, free electron lasers, or THz radiation sources have in common that they require injectors, which provide high-brilliance, high-current electron beams in almost continuous operation. Thus, the development of appropriate highly brilliant electron sources is of key importance. With its superconducting radio-frequency photo-injector (SRF gun) the Helmholtz-Zentrum Dresden-Rossendorf provided a promising approach for this key component, which has since been adopted in other laboratories. Nevertheless, some limitations occur caused by electron multipacting, which should be suppressed in order to further improve the gun. In this contribution, we present a detailed analysis of multipacting in the critical area of the SRF gun and different suppression techniques for it. The analytical predictions on the threshold for multipacting are qualitatively comparable with numerical simulation results and experimental data. Finally, we present specific surface structuring as an effective method to mitigate the multipacting phenomenon from the photocathode channel.
\end{abstract}

DOI: 10.1103/PhysRevAccelBeams.21.113402

\section{INTRODUCTION}

The evolution of theories on the multipactor effect and several experimental developments are reviewed by Kishek [1] and Chang [2] in detail. Multipacting (MP) was first observed by Gutton [3] in 1924 and further studied and described by Farnsworth [4] in 1934. Later, in the 1930s and 1940s, MP was analysed theoretically as well as experimentally for a flat gap. This led Gill and von Engel to introduce a parameter $k$ equal to the ratio of the impact velocity of the primaries to the emission velocity of secondaries that they assumed to be constant [5]. Later, this was denoted by other researchers as ad hoc assumption, see, e.g., [1]. Hatch and Williams reformulated this assumption based on other theories and their own experimental and theoretical findings, which allowed the establishment of susceptible zones for the fundamental and higher order MP modes in the 1950s [6,7]. This theory is called constant $k$ theory. In the 1980 s, the constant $k$ theory was

\footnotetext{
*eden.tulu@uni-rostock.de
}

Published by the American Physical Society under the terms of the Creative Commons Attribution 4.0 International license. Further distribution of this work must maintain attribution to the author(s) and the published article's title, journal citation, and DOI. advanced with the more realistic assumption of a monoenergetic non-zero initial velocity (equivalent to a few $\mathrm{eV}$ ) by Shemelin [8] and by Vaughan [9]. Additionally, the phase stability for the case of non-zero starting energy is introduced $[8,10]$. The latter theory also claims that the negative starting phase substantially widens the MP zone width. These concepts have been extended and theoretical and experimental studies have been carried out by many experts [11]. Most importantly, in recent decades, Furman and Pivi developed a mathematically self-consistent, phenomenological probabilistic model for the secondary emission process, which provides a very good fit to experimental data of the secondary emission yield (SEY) [12]. The model considers the probability of three kinds of emitted secondary electrons: true, backscattered, and rediffused electrons. This emission model is employed throughout our study and is explained in more detail in the next section.

Basically, the primary electrons are released from one of the structure's walls. These primary electrons, which are unwanted, are triggered by certain conducive conditions. The free electrons are accelerated by radio frequency (rf) fields and impact the surface at an angle $\left(\theta_{0}\right)$. The electrons interact with the material and its structure and transfer energy. Consequently, further electrons from inside of the material are excited and escape the surface. They are denoted as secondary electrons. In general, the multipacting 
takes place in case of the electron avalanche build up due to the enhancement of these secondary electrons.

The population of the secondary electrons is mainly determined by the surface characteristics, the impact energy of the primary electron $\left(U_{0}\right)$ and the impact angle of the primary electron $\left(\theta_{0}\right)$. This phenomenon of resonant electron multiplication may lead to a thermal breakdown [13]. The common types of MP in the literature are: onepoint (one-sided) MP, where the electron trajectories refer only to one surface of the structure, and two-point MP, where the electron hits two opposite impact sites on the same surface while two-sided MP refers to an electron hitting two different surfaces of the structure.

This paper is outlined as follows: In Sec. II, we briefly present the conditions for the multiplication of the number of electrons and the model of SEY which is used for our numerical studies. In Sec. III, we describe the definition of our model. Thereafter, in Sec. V, we discuss detailed numerical studies of MP for the given geometry, respectively. In Sec. VI, we then present the simulation results for different techniques to mitigate MP. Finally, our conclusion for MP prediction and suppression methods in the coaxial part of the SRF gun is summarized in Sec. VII.

\section{MULTIPACTING CONDITION}

There are two MP conditions for the multiplication of the number of electrons to take place by impacting the structure's wall repeatedly. First, the trajectory of the secondary electrons should fulfill specific resonance conditions in case of two-sided MP. The order of multipactor can be defined as the number of rf periods taken for the electrons to transit from the initial point until they hit the wall of the structure. The transit time of the electrons for the two-sided multipactor should be an odd integer number $(2 n-1)$ of half $\mathrm{rf}$ periods $[7,9,1,13,14]$ whereas the transit time for one-point MP is mostly determined by the electron initial longitudinal velocity and the normal electric field [1,15-17] The other criterion is that the secondary emission yield (SEY) of the impacted surface material is larger than 1. The SEY can be defined as the ratio of the emitted and incident current.

\section{A. Model of secondary emission yield}

In this paper, we assume that the initial particle is emitted from the surface of the cathode according to the Gaussian emission model with an initial particle energy of $4 \mathrm{eV}$. The emission of secondaries is governed by the advanced probabilistic emission model developed by Furman and Pivi. The model distinguishes three different ways of generating secondary electrons: elastic reflection (backscattered electrons), true secondary electrons, and inelastic reflection (rediffused electrons). These electrons are denoted as $I_{b}, I_{t s}$, and $I_{r}$, respectively. The total SEY is the ratio of the number of these three types of secondary electrons over the

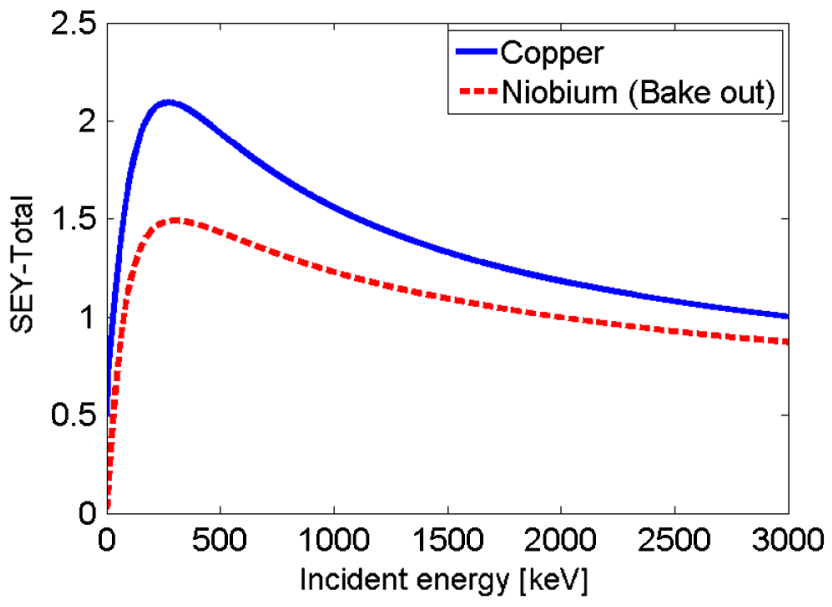

FIG. 1. The total SEY as a function of incident energy at zero incident angle for $\mathrm{Cu}$ and $300^{\circ} \mathrm{C}$ Bakeout $\mathrm{Nb}$ as used in CST STUDIO SUITE®.

number of incident electrons [12]. Obviously, considering all three types of secondary electrons is very important to make a reliable multipactor prediction. Figure 1 shows the total SEY curves for a copper $(\mathrm{Cu})$ cathode and a niobium $(\mathrm{Nb})$ cavity as a function of impact energy as used in our simulation. MP is generally expected where the SEY is greater than one between the first crossover of the energy $\left(U_{c 1}\right)$, and the second crossover of the energy $\left(U_{c 2}\right)$, e.g., MP is expected between 27.3 and $3019 \mathrm{eV}$ for our SEYmodel of $\mathrm{Cu}$. The maximum generation of secondaries is expected at the peak SEY $\left(\delta_{\max }\right)$ for perpendicular incidence with the corresponding energy $\left(U_{\max }\right)$.

\section{GEOMETRY DESCRIPTION}

A superconducting radio frequency (SRF) gun has been in operation at Helmholtz-Zentrum Dresden-Rossendorf (HZDR) since 2007. The SRF gun is the optimum choice for the ELBE superconducting (SC) linac for the following reason: the most important feature of the SRF gun is that it operates in a continuous wave $(\mathrm{CW})$ mode with low power consumption. It is also possible to overcome all the drawbacks of the other two types of $\mathrm{CW}$ guns; the $\mathrm{DC}$ photo guns and normal conducting (NC) rf photo gun. A detailed comparison of these guns has been documented in $[18,19]$. However, the SRF gun is subjected to electron MP. Due to this effect, it turns out that a strong input mismatch paired with a resonance drift makes it infeasible to raise the accelerating gradient by increasing the incident rf power. Therefore, this phenomenon is a serious problem that limits the accelerating gradient in the cavity to very low values [20]. Hence, minor design changes are under investigation that should allow the SRF gun to operate up to the desired electric field strength [19].

In different stages of our numerical studies, we regarded different simplifications of the complete model of ELBE's SRF gun. We refer to three different models in this paper such as a complete model of the SRF gun (model A), the 


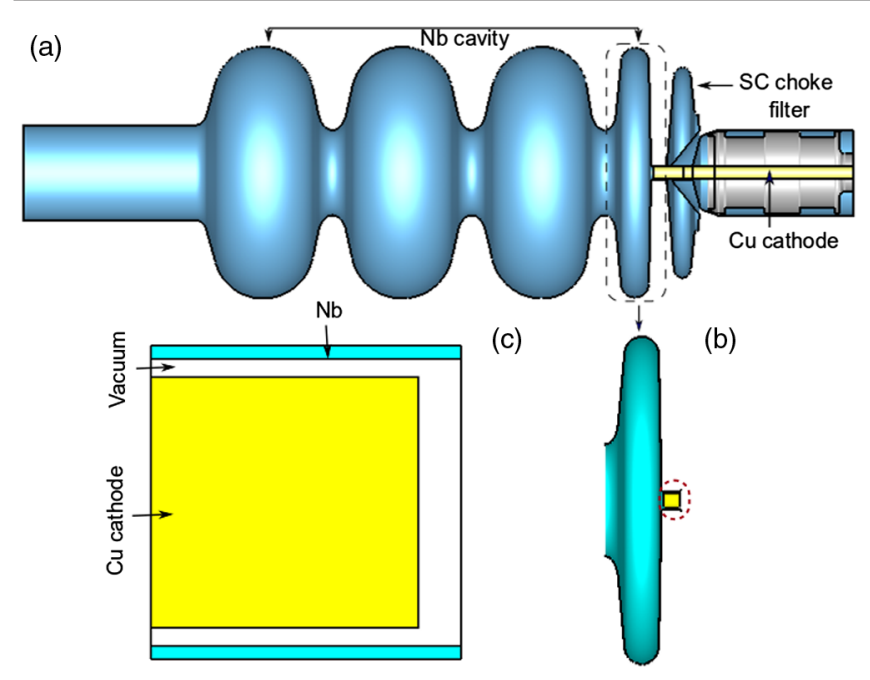

FIG. 2. (a) A complete CST MICROWAVE STUDIO® (CST MWS) model of the SRF gun (model A) that comprises a $1.3 \mathrm{GHz} 3.5$ cells TESLA-like Nb cavity, a NC photocathode, and a SC choke filter; (b) the coaxial structure (marked in red) plus half-cell cavity structure (model B); (c) a simplified model covering just the coaxial part of the cathode region (model $\mathrm{C}$ ) that is most relevant with respect to MP. The inner material is $\mathrm{Cu}$ (in yellow) and the outer is $\mathrm{Nb}$ (in blue-green).

coaxial structure plus half-cell cavity (model B) and just the coaxial part of the SRF gun (model C). Figure 2 illustrates model A that comprises a superconducting $1.3 \mathrm{GHz} 3.5$ cell TESLA-like Nb cavity, a NC photocathode and a SC choke filter. The NC photocathode is inserted at the circular end of the $\mathrm{Nb}$ half-cell cavity with a $0.75 \mathrm{~mm}$ vacuum gap for thermal and electrical insulation. The aforementioned choke filter surrounds the photocathode in order to prevent a leakage of the rf field from the cavity. In this paper, we focus on the results of the $\mathrm{Cu}$ cathode, i.e., of model $\mathrm{C}$, with a $\delta_{\max }$ of 2.1 at $250 \mathrm{eV}$ impact energy and the $\mathrm{Nb}\left(300^{\circ} \mathrm{C}\right.$ Bakeout) cavity with a $\delta_{\max }$ of 1.49 at $300 \mathrm{eV}$ impact energy. We also considered different anti-MP layer materials. These investigations will be published in a separate paper. The design of the complete ELBE SRF gun is discussed in detail elsewhere [18].

The cathode region is the most critical part with respect to MP. Therefore, this region is of main interest when aiming to reduce MP and it is reasonable to confine the simulation domain to it. This also allows the employment of a sufficient mesh resolution for the MP simulation while keeping the computational effort low enough for systematic optimization studies under various aspects. Thus, our further study considers mostly model $\mathrm{C}$. In the next sections, we will present and validate the numerical and analytical results of this model.

\section{NUMERICAL STUDY ON MULTIPACTING IN SRF GUN}

Generally, there are three steps for an MP simulation: The first step is the geometry definition and the calculation of the electromagnetic field for the given geometry. The second step is calculating and tracking the motion of a large number of particles in the electromagnetic field distribution along the structure. Finally, the third step is identifying a possible MP behavior in the collection of particle tracking data. All three steps can be done using CST STUDIO SUITE® [21]. Other features of this software worth mentioning include the availability of numerous data in the material library, an advanced probabilistic emission model and an adequate post-processing of CST PARTICLE STUDIO ${ }^{\circledR}$ (CST PS). Therefore, we chose the Particle-inCell (PIC) solver of CST PS for our numerical studies on MP. In the next sections and subsections, we will present a detailed description and our results. Additionally, there are recent MP studies on low beta spoke cavities [22,23] and coaxial waveguides [24] using CST PS that provide detailed technical procedures.

\section{A. Electromagnetic field calculation}

An MP analysis requires an rf field simulation in order to track the motion of the electron under the electromagnetic force. We computed the operating frequency and the corresponding electromagnetic (EM) field pattern for model B and model C (see Fig. 3) using the eigenmode solver and the frequency domain (FD) solver of CST MWS, respectively. In order to calculate the EM field values in the computational domain, the boundary conditions need to be specified at the boundary surface [25]. Appropriate boundary conditions and sufficient mesh resolution of the simulated model are significant in order to get accurate results. To achieve a sufficient field resolution, which is required for our particular interest of MP study, we employed local mesh refinement around the cathode region. We specified perfect electric boundary condition (PEC) at the surface and perfect magnetic boundary condition (PMC) at the end of the structure. We applied two symmetry planes modeled by magnetic boundary
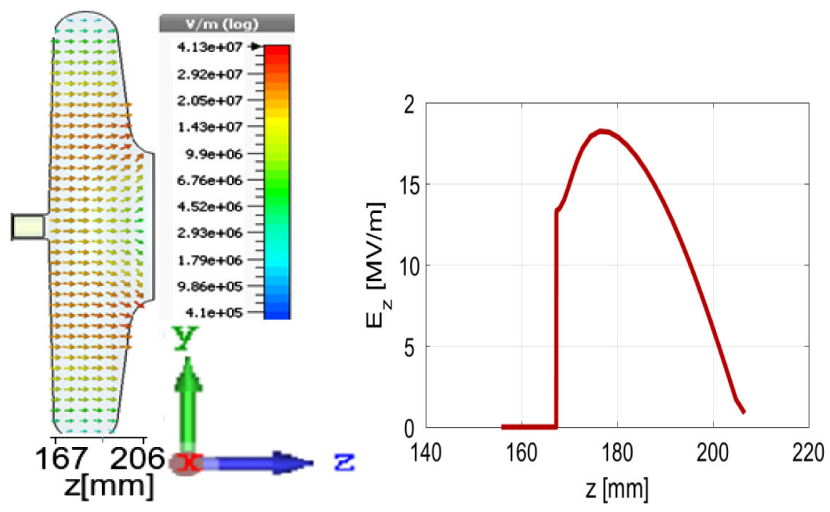

FIG. 3. Electric field distribution in model B consisting of the coaxial line plus the half-cell cavity at $1.3 \mathrm{GHz}$. Left: Distribution of the electric field in the half-cell cavity; Right: Axial electric field $E_{z}$. 


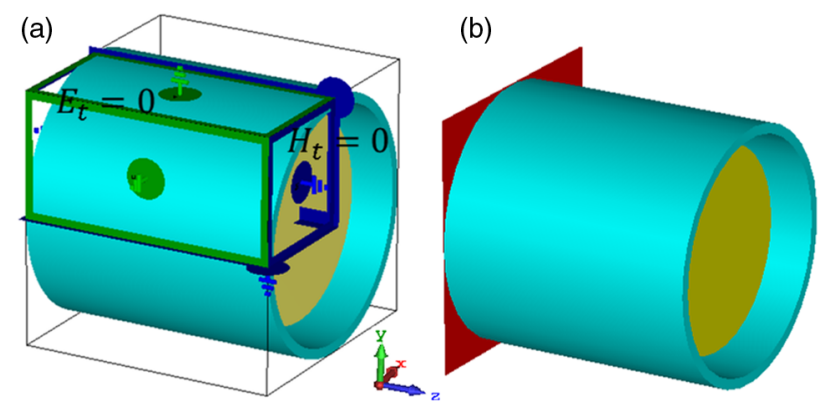

FIG. 4. (a) The PEC (green) and PMC (blue) are specified on the boundary and the symmetry plane. (b) A waveguide port (in red) is used as excitation source.

conditions in order to reduce the computational time by a factor of four and thus, in turn, to increase the accuracy.

We computed the distribution of the electric and magnetic field in model B using the eigenmode solver of CST MWS. The SRF gun operates with the $\mathrm{TM}_{010}$ mode at $1.3 \mathrm{GHz}$. The peak electric field on axis is $18.3 \mathrm{MV} / \mathrm{m}$, which is normalized by $1 \mathrm{~J}$ in the eigenmode solver, whereas the highest field in the half cell is about $41 \mathrm{MV} / \mathrm{m}$. The computed resonant frequency is $1.299 \mathrm{GHz}$. The electric field distribution and the axial field $E_{z}$ are displayed in Fig. 3. In order to track the trajectories of the particles in the field, we imported the calculated EM field into the PIC solver of CST PS. In the PIC solver, the field amplitude was scaled by using a scaling factor $a m$ to achieve the desired rf field for the MP simulation. Therefore, we carried out a field scan in order to obtain the field range in which MP exists around the cathode.

As mentioned previously, we further simplified the model into model $\mathrm{C}$ [see Fig. 2(c)] that just comprises the cathode part of the SRF gun in order to reduce the computational cost and to enable a detailed investigation of MP, which requires a very dense mesh. We applied the FD solver of CST MWS to calculate the electromagnetic fields utilizing a waveguide port shown in Fig. 4(b). Based on the boundary conditions shown in Fig. 4(a), one obtains a standing wave field in the coaxial line structure of model C with a maximum electric field of $15.03 \mathrm{kV} / \mathrm{m}$ for an input power at the waveguide of $1 \mathrm{~W}$. The field pattern and the small gap width provide favorable conditions for MP. Finally, we imported the calculated EM fields (see Fig. 5) into the PIC solver of CST PS to track the particles.

\section{B. Multipacting simulations}

Practically, the rf field leaking out of the cavity into the photocathode region is high enough to initiate MP around the photocathode of the SRF gun. Indeed the experimental data gained at ELBE as well as the theoretical solution for MP prediction confirmed its susceptibility in the vicinity of the cathode. Thus, in order to better understand the MP behaviour and clearly identify the range of the electric field

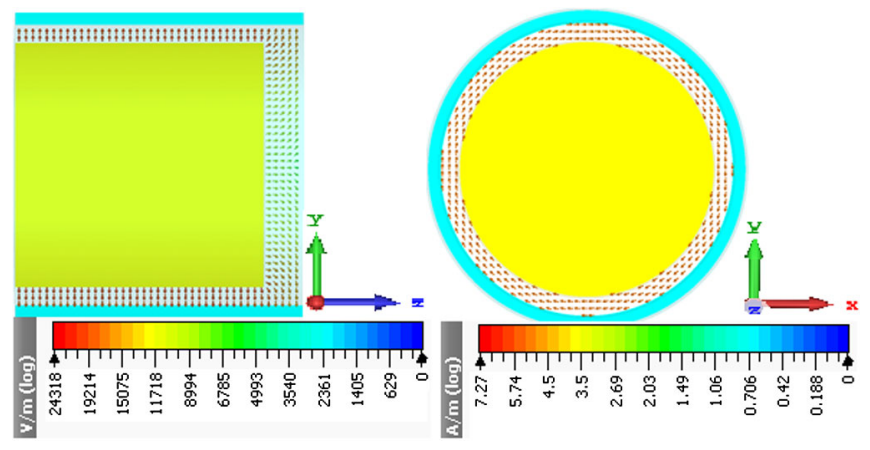

FIG. 5. Electromagnetic field in the coaxial line structure at $1.3 \mathrm{GHz}$. Left: Distribution of the electric field; Right: Distribution of the magnetic field.

in which it occurs, we performed an intensive simulation study with realistic assumptions. In this section, we present simulation results and analyse MP characteristics (e.g., type of MP determined from the trajectories of the electrons and the field level).

The number of particles over time was evaluated in the simulated model (model C) as shown in Fig. 6 using CST PS. Usually, a growth in this number as a function of time is a characteristic for electron MP. The trajectories of these particles are also depicted in Fig. 7. Furthermore, CST PS generates emission and collision currents for each separate surface. These data enable us to calculate the average SEY $\left(\mathrm{SEY}_{a}\right)$ [22] that is defined as the integral of the emission current divided by the integral of the collision current. The calculation is made for inner and outer surfaces separately. From this, we evaluated MP probability and determined the field level at which MP occurs. The following assumptions were made for the initial particle: (i) emission according to the Gaussian emission model, (ii) an energy of a few $\mathrm{eV}$ $(0-4 \mathrm{eV})$, (iii) emission during the first period of the rf cycle. Figure 5 shows that the transverse electric and magnetic field are predominant in the gap between the inner and outer conductor where the MP is critical. The initial

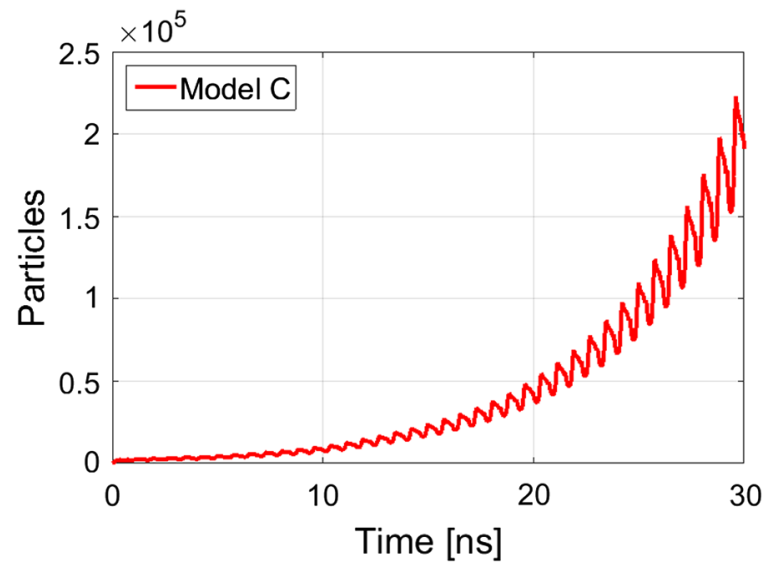

FIG. 6. Exponential growth of particles in the cathode region at $127.7 \mathrm{kV} / \mathrm{m}$. 


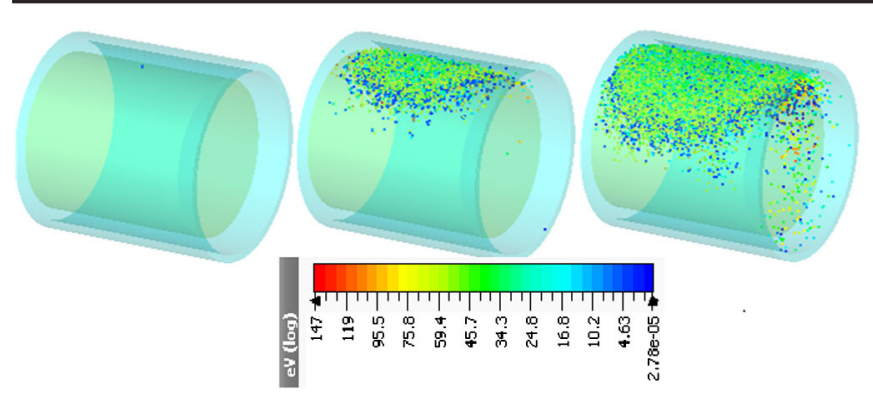

FIG. 7. Development of Multipacting in time in Model $\mathrm{C}$ at $127.7 \mathrm{kV} / \mathrm{m}$ after $0.2 \mathrm{~ns}$ (left), after $13.4 \mathrm{~ns}$ (middle) and after $25.7 \mathrm{~ns}$ (right).

electrons travel towards the opposite wall where they produce a number of secondary electrons. The generation of these secondaries is governed by the advanced probabilistic emission model by Furman and Pivi [12] as implemented in CST PS. Moreover, there are a number of emissive materials in the material library of CST STUDIO SUITE®. Among them, as shown in Fig. 1, Cu was used for the inner surface (the cathode) and $300^{\circ} \mathrm{C}$ Bakeout $\mathrm{Nb}$ for the outer surface. Furthermore, in order to minimize computational time, the space charge effects were neglected in the PIC simulation as recommended in $[26,27]$. Since the susceptibility region was already known, a particle source is assigned to a single point on the cathode using the Gaussian model. This type of initial source is denoted as "particle point source" in CST PS. The point sourcelike assumption was reasonable since it is well known that even the appearance of a single particle at the right time can initiate MP. Nevertheless, in order to check for the existence of stochastic fluctuation in the results (if any), we also ran a simulation with an area source covering the complete inner conductor. Since both results agreed very well, and to save simulation time, we decided to use the particle point source throughout all the following simulations. The next step was the comparison of the results from model B vs model C in order to validate the latter structure. For this, the calculated electromagnetic field of model B (see Fig. 3) was imported into CST's PIC solver and scaled to obtain the field range in which MP occurs. The scaling factor was determined such that the electric field in the gap varied from 45 to $255 \mathrm{kV} / \mathrm{m}$. This range corresponds to a cavity field in the range of 0.45 to $3.69 \mathrm{MV} / \mathrm{m}$. Figure 8 illustrates that the peak $\mathrm{SEY}_{a}$ was obtained at the gap field of $112.6 \mathrm{kV} / \mathrm{m}$. The corresponding electric field in the half-cell cavity for that case was $1.55 \mathrm{MV} / \mathrm{m}$ which is in good agreement with experimental data [28]. In the same figure, the $\mathrm{SEY}_{a}$ curve (in red) for model C shows that the field level of the gap field is in the same range although the field interval with MP is somewhat narrower. Since the values close to the maximal SEY ${ }_{a}$ were similar, it is reasonable to consider only the coaxial part of the structure, i.e., model $\mathrm{C}$, for further investigation. Figure 9 shows the simulation result for $\mathrm{Cu}$ (inner cathode surface) and $\mathrm{Nb}$ (outer surface) for model $\mathrm{C}$

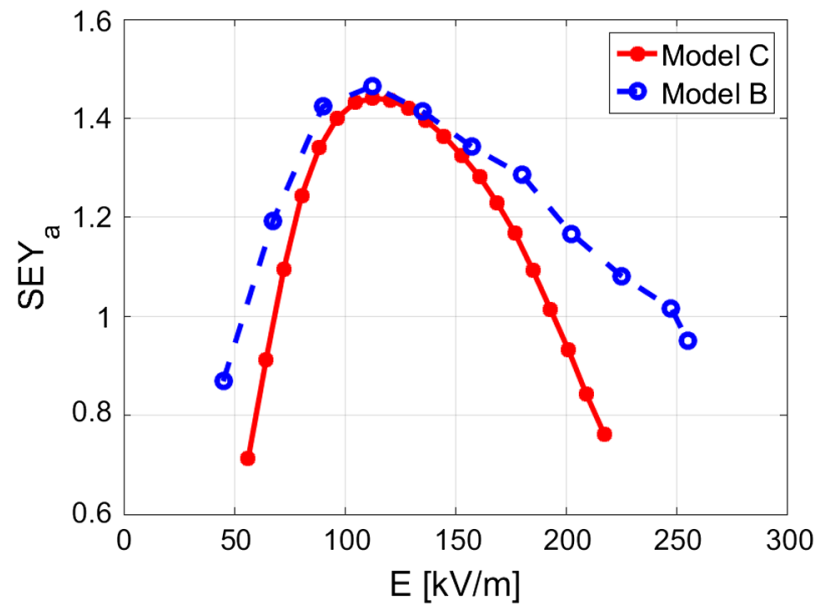

FIG. 8. Comparison of the two simulation models, model B and model C. SEY curves vs electric field strengths are shown for the $\mathrm{Cu}$ surface. The electric cathode field is used for model $\mathrm{C}$ while the on-axis field is considered for model B.

with a vacuum gap width of $0.75 \mathrm{~mm}$. The $\mathrm{SEY}_{a}$ curve for Cu shows that the lower limit $E_{\min }$ and the upper limit $E_{\max }$ of the electric field amplitude were $\sim 70$ and $\sim 194 \mathrm{kV} / \mathrm{m}$, respectively, to allow an electron to gain sufficient energy for MP. SEY ${ }_{a}$ was larger than one for $U_{a}$ values between 35 and $50.8 \mathrm{eV}$. As already noted above, the peak $\mathrm{SEY}_{a}$ of 1.44 was produced at the gap electric field of $112.7 \mathrm{kV} / \mathrm{m}$. More importantly, the analytical prediction of the field level and the impact energy for 1st order two-point MP in a vacuum gap width of $0.75 \mathrm{~mm}$ (see Table I in Appendix A) were within the MP limits obtained by numerical simulation.

Similarly, the $\mathrm{SEY}_{a}$ for the $\mathrm{Nb}$ surface was calculated in order to obtain the MP threshold. As Fig. 9 depicts, for a vacuum gap width of $0.75 \mathrm{~mm}$, the $\mathrm{SEY}_{a}$ of $\mathrm{Nb}$ was below 1 in the whole range of field levels. Thus, the contribution

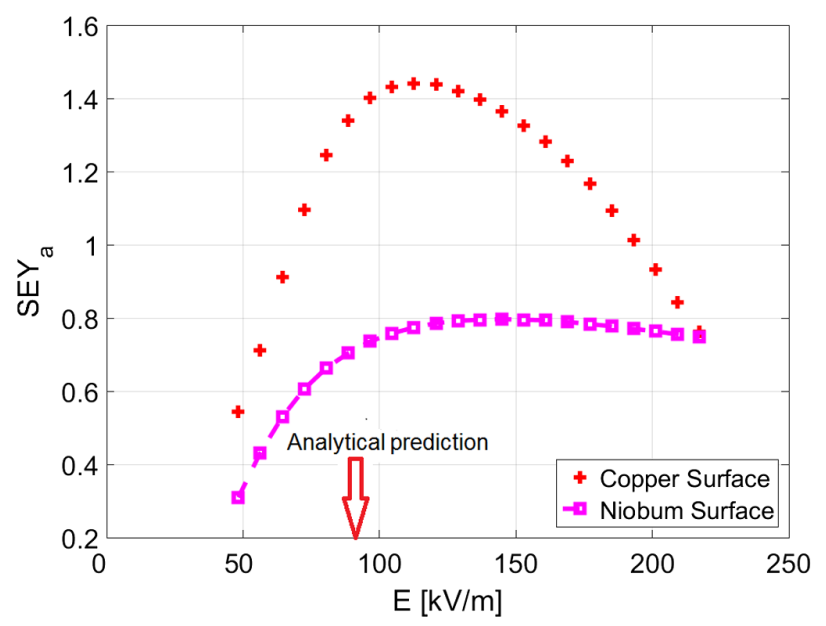

FIG. 9. $\mathrm{SEY}_{a}$ as a function of electric field strength for $\mathrm{Cu}$ and $\mathrm{Nb}$ surfaces for a gap width of $0.75 \mathrm{~mm}$. The analytical prediction of the field level causing MP is indicated by the red arrow. 
of the $\mathrm{Nb}$ surface to MP was negligible. Figure 9 serves as a reference for comparison with different results in the sections below.

\section{MULTIPACTING SUPPRESSION TECHNIQUES}

In the last decade, several approaches were proposed and implemented to mitigate MP phenomena for different $\mathrm{rf}$ components. For example: rf conditioning of the components [29]; anti-MP coatings, e.g., TiN, to introduce low surface conductivity [30]; treatment of metal surfaces by pulsed-laser irradiation [31]; surface cleaning [32]; choosing geometries that are less susceptible to MP, e.g., elliptical cavity shapes $[33,34]$; geometry modifications; static perturbation (applying DC electric and magnetic field on the surface) [35,36], blue [37,38]; antimultipactor grooves, e.g., rectangularly and triangularly grooved surfaces. The latter technique is effective to reduce $\mathrm{SEY}_{a}$ below the MP threshold in dipole and wiggler magnets [39]. Moreover, theoretical and experimental studies show that changing surface profiles (surface-grooving) in different $\mathrm{rf}$ structures can also suppress MP and improve the breakdown threshold for vacuum multipactor [40-44]. Hence, we have investigated the following remedies for our model to check if these allow the MP conditions to be broken down: DC biasing voltage (static electric field perturbation), geometric modification (frusto-conical cathode, varying the diameter of the outer conductor), antimultipactor grooves of the cathode's surface (rectangular, sawtooth, isosceles), and anti-MP coatings. According to the scaling law for the MP threshold, which was carefully studied in [35], MP may be cured by modifying the dimensions of the inner and outer conductors of the coaxial line. However, it might be infeasible to implement this method directly in our case because the geometry of the SRF gun cannot be changed. Instead, only the diameter of the outer conductor as well as the cathode (frusto-conical shape) itself have been investigated. The first one will be discussed in the following section while the latter one is published elsewhere [45], where we observed that the frusto-conical cathode shape does not suppress MP in our case. Additionally, we present and discuss the result of DC biasing voltage (static electric field perturbation), radial rectangularly grooved surface and isosceles triangularly grooved surface.

\section{A. DC biasing (static electric perturbation)}

The idea of applying a DC bias voltage is to perturb the resonant conditions of the trajectory by developing a radial repelling force. A detailed analysis of this method to suppress MP was discussed in $[35,46]$. In a coaxial line structure, the optimal suppressing DC voltage for the standing wave, according to [46], can be set by

$$
E_{D C}(r, z)=\frac{V}{\ln \frac{r_{\text {out }}}{r_{\text {in }}}} \frac{1}{r}
$$

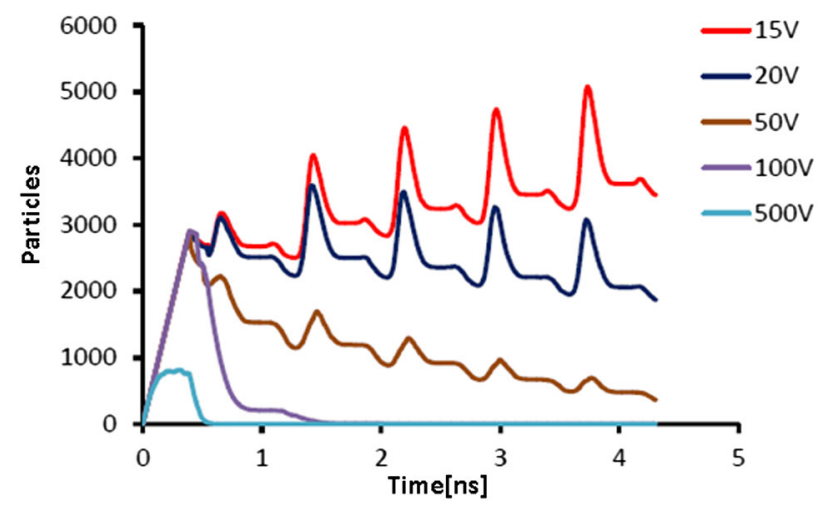

FIG. 10. Number of particles as a function of time for various DC bias voltages. MP can be totally suppressed in case of 500 and $100 \mathrm{~V}$.The simulation was performed using model B.

where $r_{\text {out }}$ and $r_{\text {in }}$ are the outer and the inner radii of the structure and $(r, z)$ are the radial and longitudinal coordinates of a field point in a cylindrically symmetric structure. The suppressing DC voltage $(V)$ obeys the following general scaling law [35]:

$$
V \sim f Z d_{\text {out }},
$$

where $f$ is the frequency, $Z$ is the impedance of the line, and $d_{\text {out }}$ is the outer conductor diameter. The simulation was performed using model B in two ways: defining the voltage directly at the cathode or importing the electrostatic field after computing it separately. In both cases, the results were similar. We varied the suppressing DC voltage in the range of 15 to $500 \mathrm{~V}$. As shown in Fig. 10, MP was totally suppressed in the case of $100 \mathrm{~V}$ and above but even $50 \mathrm{~V}$ could be sufficient. However, in practice it has been found that a voltage up to $7 \mathrm{kV}$ was required to suppress MP reliably [20]. Therefore, further investigations are needed to match numerical and experimental data.

\section{B. Antimultipactor grooved surface}

Beside various techniques to suppress MP, grooving the surface has demonstrated success for different structures as described in several papers. Antimultipactor grooves were first proposed in [42] and some results are presented there. In our studies, we investigated numerically rectangular and triangular surfaces for the coaxial line structure, i.e., model $\mathrm{C}$, as the critical part of the studied SRF gun. We considered the $\mathrm{Cu}$ material of the inner surface and the $\mathrm{Nb}$ material of the outer surface with a peak SEY of 2.1 and 1.4 , respectively, for the simulation of an antimultipactor grooved surface. Because of practical reasons, we applied the grooving only on the inner part of the coaxial line structure.

The basic principle of a grooved surface to reduce MP is to disturb the resonant condition by altering the trajectories of the electrons and by that diminishing the transit time to less than half an rf period. Figure 11 displays a graphical 

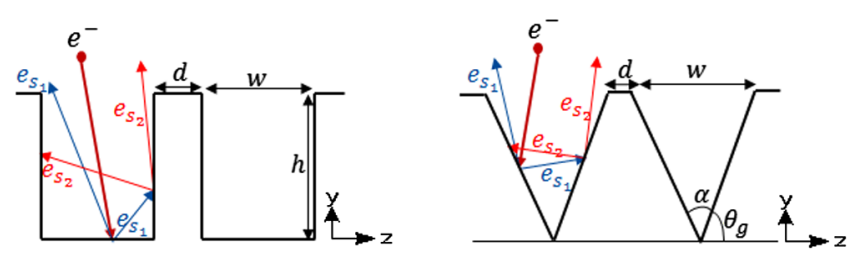

FIG. 11. Left: Rectangularly grooved surface; Right: Isosceles triangularly grooved surface $\left(\theta_{g}+\frac{\alpha}{2}=\frac{\pi}{2}\right)$.

sketch of the motion of exemplary secondaries in the grooved surface. An electron $e^{-}$enters this surface and hits the wall with a certain impact angle. Thus, the first secondaries $\left(e_{s_{1}}\right)$ are produced. Some of these secondaries might escape the groove depending on the impact angle. The others hit the opposite side of the wall, where either they might be absorbed or a second generation electron $\left(e_{S_{2}}\right)$ might be produced. This process is repeated until the energy of the higher generations becomes too low and all secondaries are absorbed by the surface [40]. In general, most secondaries are unlikely to survive after multiple collisions. Since our studies revealed that a grooved structure is a promising suppression technique for the SRF gun, we optimized the model. Initially, we varied the geometry parameters of the grooves manually with respect to the number of particles over time. Based on these results that are published elsewhere [47], we could identify geometry parameters with a strong impact on MP. In order to obtain the global optimum for our models, these parameters were then further optimized using the so-called trust-region algorithm [48-50]. The original objective function $\min _{x \in X} f(x)$, where $X \subset R^{n}$, is approximated either by a quadratic or a linear model. In CST PS, the linear model function $m_{k}(s)$ is used to approximate $f(x)$. With a given initial point, the $m_{k}(s)$ builds up in a "trustregion" in which we trust our model. In case of our optimization problem, the initial parameter values and the initial parameter bounds (initial trust-region radius $\Delta_{k}$ ) were chosen based on our previous work mentioned above. The algorithm solves the trust-region subproblem in order to determine a candidate trial step $\left(s_{k}\right)$. Then, if the solution indicates an acceptable reduction of the goal function, the radius of the trust-region will be updated (increased) for the next evaluation. This will be repeated until convergence. The basic algorithm is stated in Appendix B. In general, the algorithm can be chosen as a global or local optimization in CST PS.

In contrast to the preliminary studies, this was done for both the $\mathrm{Cu}$ and the $\mathrm{Nb}$ surface with respect to the more suitable $\mathrm{SEY}_{a}$ and no longer to the number of particles. The initial parameters were chosen based on the manual results and the optimization goal function requires the $\mathrm{SEY}_{a}$ to be smaller than the MP threshold $\left(\mathrm{SEY}_{a}<1\right)$. Even though a rather powerful computer (Intel® Xeon® E5 $3.30 \mathrm{GHz}$ $256 \mathrm{~GB}$ ) was used for our optimization, it still takes around three hours of computational time for one iteration of the
PIC solver due to the high number of mesh cells (approximately 10 million) required for the grooved surface. As already noted, the higher generations of secondaries do not contribute much to the $\mathrm{SEY}_{a}$. Thus, in order to reduce the computational time, the $\mathrm{SEY}_{a}$ was calculated for the first few generations of secondaries only (e.g., $5 \mathrm{~ns}$ ).

\section{Cylindrically symmetric rectangularly grooved surface}

The rectangularly grooved surface is characterized by the following geometrical parameters: the width of the groove $(w)$, the depth $(h)$, and distance $(d)$ between the grooves. The rf field at $1.3 \mathrm{GHz}$ was calculated for the model with $0.75 \mathrm{~mm}$ gap width using the FD solver of CST MWS. The grooved surface requires a very dense mesh to obtain a field map with sufficient accuracy. The distribution of the electric field in the rectangularly grooved model is shown in Fig. 12. The electrons gain energy from the electric field. The grooved surface provides an attenuated amplitude of the electric field resulting in a remarkable reduction of the electron energy. However, more importantly, it limits the electron transit distance because many of the electrons impact the walls of the grooves several times during one rf half cycle. Thus, the resonant condition mentioned in Eq. (3) (see Appendix A) is not fulfilled and the $\mathrm{SEY}_{a}$ is reduced considerably.

The SEY ${ }_{a}$ and the impact energy for $\mathrm{Cu}$ and $\mathrm{Nb}$ surfaces for the rectangularly grooved model are illustrated in Fig. 13. In combination with the already mentioned optimization algorithm, the values for the rectangular surface were found to be $w=0.23 \mathrm{~mm}, h=0.80 \mathrm{~mm}$

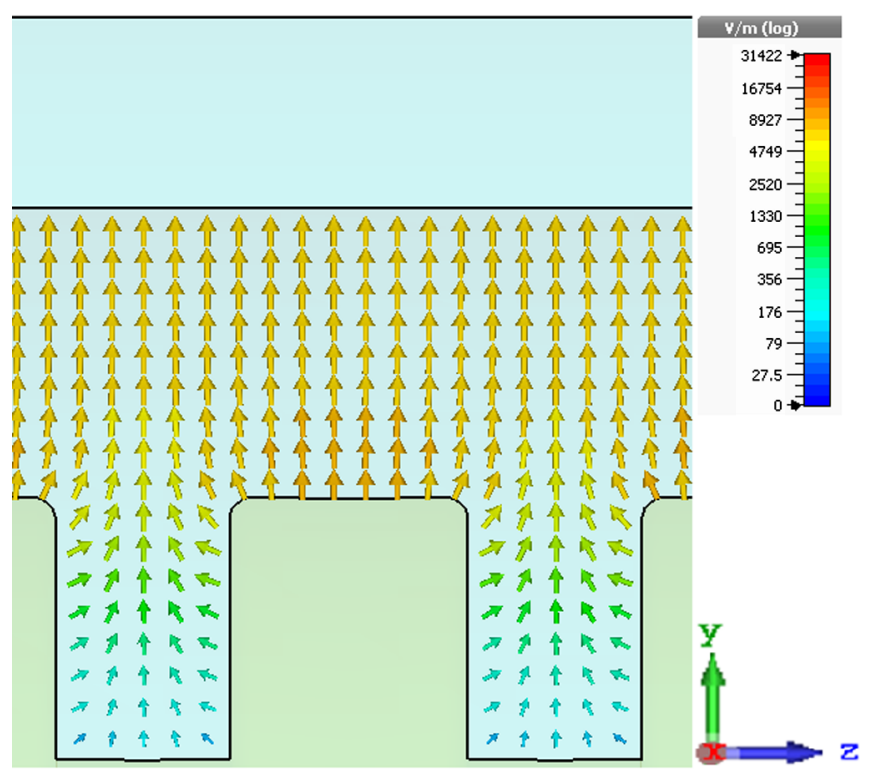

FIG. 12. Electric field distribution inside of the rectangularly grooved model with the surface parameters of $w=0.23 \mathrm{~mm}$, $h=0.80 \mathrm{~mm}$, and $d=0.48 \mathrm{~mm}$. The radius of the rounded corner is considered to be $0.05 \mathrm{~mm}$. 
and $d=0.48 \mathrm{~mm}$. The amplitude of the electric field was swept in the range of 91.76 to $250.6 \mathrm{kV} / \mathrm{m}$ in order to obtain the limits for MP. This result shows that the $\mathrm{SEY}_{a}$ of $\mathrm{Cu}$ remains slightly below or near to one above $124 \mathrm{kV} / \mathrm{m}$. The same figure also displays the result of our simulations for flat $\mathrm{Cu}$ and $\mathrm{Nb}$ surfaces for reference. The peak $\mathrm{SEY}_{a}$ of the grooved $\mathrm{Cu}$ surface is abated from 1.440 to 0.956 at $112.7 \mathrm{kV} / \mathrm{m}$ where the maximum generation of secondaries was observed in the flat gap. The corresponding $U_{a}$ for $\mathrm{Cu}$ is $21.1 \mathrm{eV}$, i.e., lower than the $U_{c 1}$ of $27.3 \mathrm{eV}$. In the case of $\mathrm{Nb}$, the flat and grooved surfaces have shown similar curves of $\mathrm{SEY}_{a}$ since grooving is implemented only on the $\mathrm{Cu}$ surface. Figure 13(a) shows that all values of the average SEYs of $\mathrm{Cu}$ for the rectangularly grooved model stayed near to the threshold of 1 in the entire range of the electric field strength. Hence, the rectangular grooving is effective in reducing the peak $\mathrm{SEY}_{a}$ for the $\mathrm{Cu}$ surface and suppressing MP in the cathode unit.

(a)

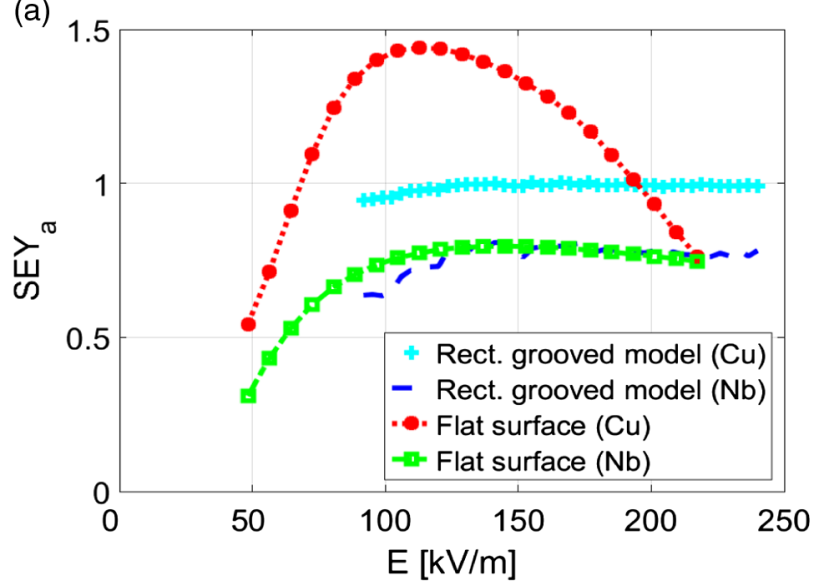

(b)

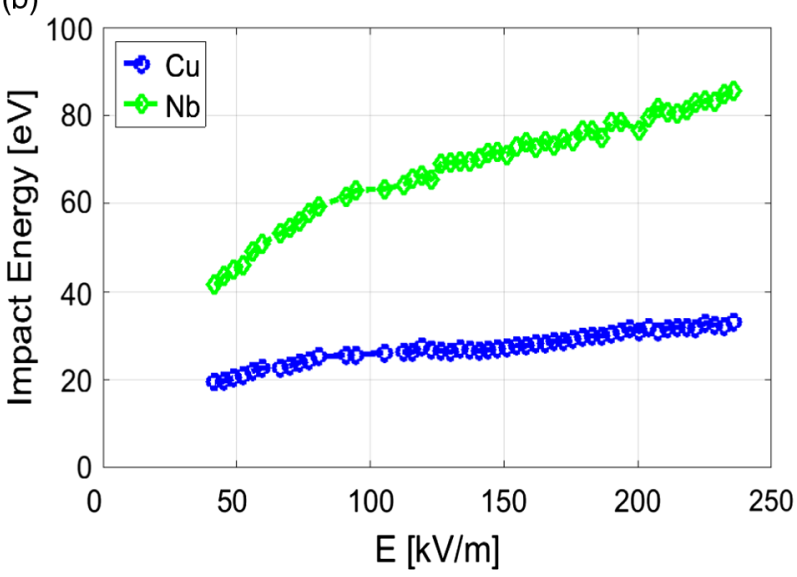

FIG. 13. (a) Comparison of the average SEY for the model shown in Fig. 12 with that of a flat surface (model C). The former one reduces the average SEY to values below 1; (b) The impact energy of the resonant trajectories in the rectangularly grooved surface (shown in 12).

\section{Isosceles triangularly grooved surface}

Next, we investigated the influence of isosceles triangular grooves constructed from two tilted surfaces with the same grazing angle $\theta_{g}$ (see Fig. 11). Such grooves can be mechanically fabricated either by rolling or by extrusion. In our simulation, the groove tips were rounded with radius of $0.05 \mathrm{~mm}$ since manufacturing of sharp tips is expensive $[39,40]$ and, more importantly, rounding avoids field enhancement. The groove width $w$, and grazing angle $\theta_{g}$ were varied in the simulation study. Previous studies [40,42] revealed that MP is sensitive to the angle $\alpha$ and the grazing angle $\theta_{g}$ but not to the size of the triangular grooves. However, the size should be comparable to the penetration depth of the incident electrons in the metal.

The distribution of the electromagnetic field that was imported to the PIC solver is illustrated in Fig. 14. The optimum parameters for the isosceles triangles are $\theta_{g}=$ $80.7^{\circ}$ or $\alpha=18.6^{\circ}$ and $w=1.15 \mathrm{~mm}$. In order to obtain the MP limits, a parameter sweep of the electric field amplitude was performed from 78.6 to $250.6 \mathrm{kV} / \mathrm{m}$. The simulation results revealed that $\mathrm{SEY}_{a}$ slightly increases with an increase in electric field and stays constant near or at the threshold after $180 \mathrm{kV} / \mathrm{m}$ as depicted in Fig. 15(a). The $\mathrm{SEY}_{a}$ curves for a flat surface are shown in the same figure as a reference. The grooved model reduces the peak $\mathrm{SEY}_{a}$ for $\mathrm{Cu}$ and $\mathrm{Nb}$ to 0.91 and 0.24 , respectively, at $112.7 \mathrm{kV} / \mathrm{m}$ where the maximum generation of secondaries was observed in the flat gap. The corresponding $U_{a}$ (see 15(b)) are 15.60 and $28.54 \mathrm{eV}$ for both, $\mathrm{Cu}$ and $\mathrm{Nb}$, which is significantly lower than the $U_{c 1}$ of $27.3 \mathrm{eV}$ for $\mathrm{Cu}$ and $76 \mathrm{eV}$ for $\mathrm{Nb}$, respectively. In general, the isosceles

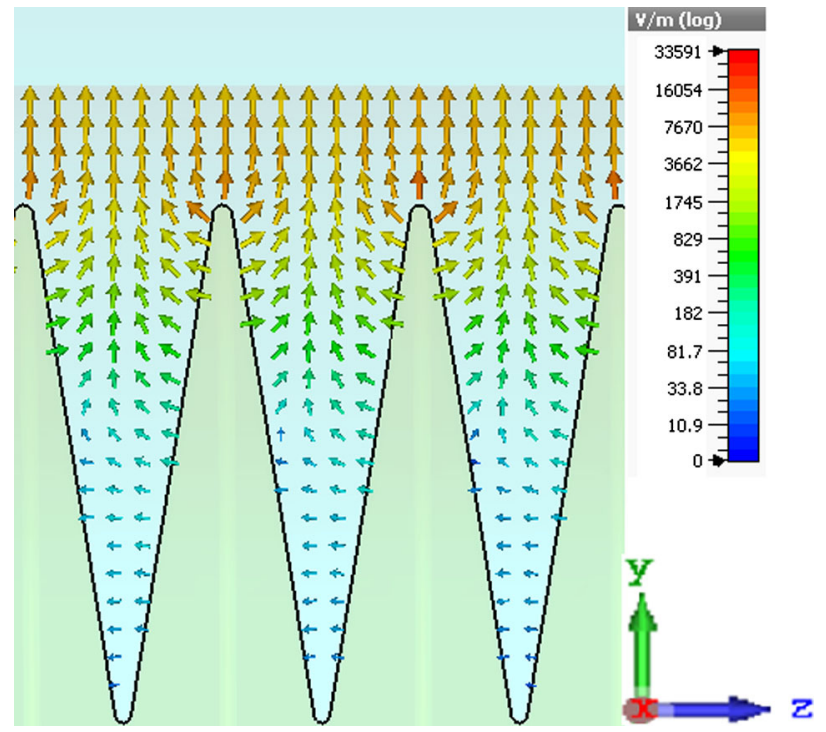

FIG. 14. Electric field distribution inside of the isosceles triangularly grooved surface. The parameters of the groove surface are $\theta_{g}=80.7^{\circ}$ or $\alpha=18.6^{\circ}$ and $w=1.15 \mathrm{~mm}$ with a radius of the round $0.05 \mathrm{~mm}$. 

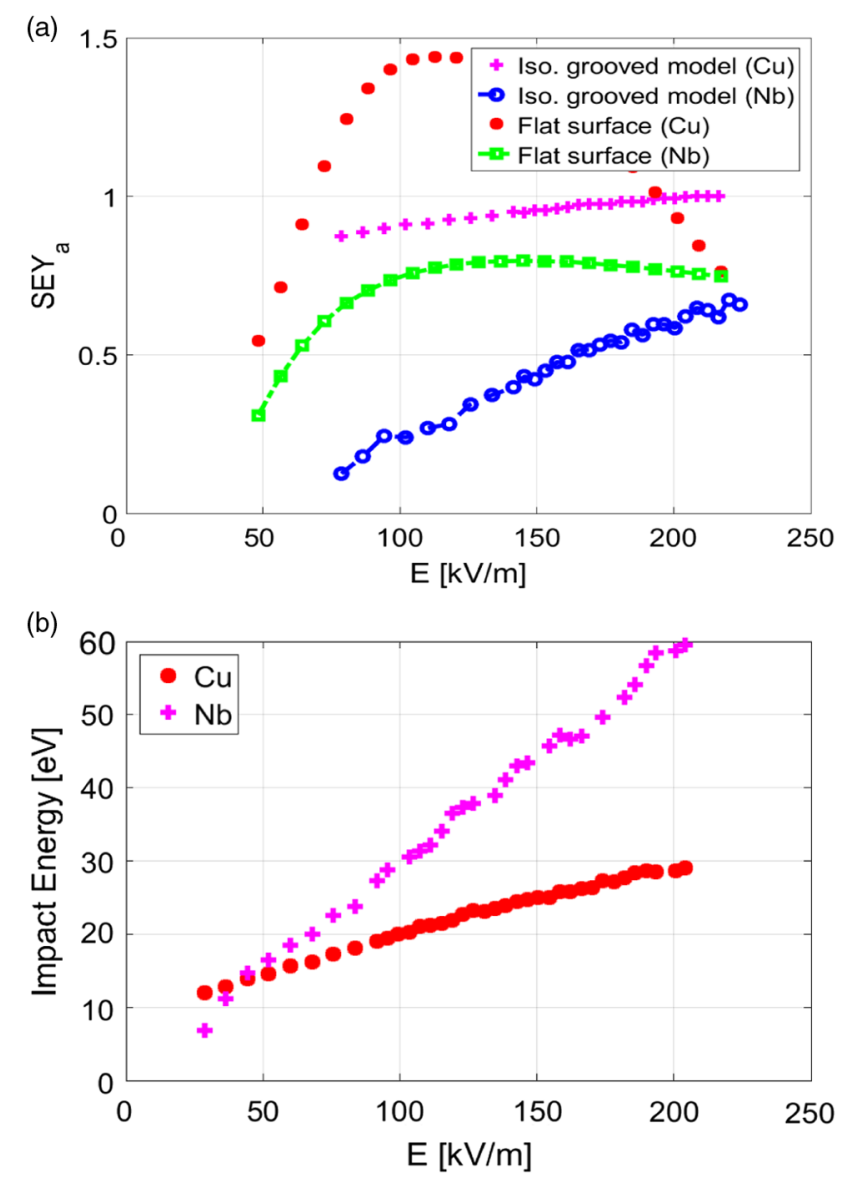

FIG. 15. (a) Comparison of the isosceles triangularly grooved model with a flat surface (model $\mathrm{C}$ ). The isosceles surface reduces the average SEY below the threshold; (b) The impact energy of the resonant trajectories in the isosceles grooved surface.

triangular surface provides a significant reduction in $\mathrm{SEY}_{a}$ and therefore MP is totally suppressed in the region.

\section{Geometry modification}

A larger gap between choke and half-cell should be advantageous to improve the cleaning of the choke filter. This becomes necessary because the cavity gradient is limited, which is probably because of particle contamination from the choke. Therefore, we numerically investigated the MP behavior of a larger gap based on the theoretical predictions (see Appendix A). The computations have been performed for gap widths $d$ of $0.75,1.5$, and $3 \mathrm{~mm}$ by changing the radius of the outer conductor. The inner radius of the cathode was kept constant at $5 \mathrm{~mm}$.

For the following comparison of the three models, only the results for $\mathrm{Cu}$ (cathode surface) are discussed in the sense of a worst case study due to its larger $\mathrm{SEY}_{a}$. The simulation result for $1.5 \mathrm{~mm}$ (see Fig. 17) shows that the peak $\operatorname{SEY}_{a}(\sim 2.1)$ was generated at $165.5 \mathrm{kV} / \mathrm{m}$ with a corresponding impact energy of $162 \mathrm{eV}$. $E_{\min }$ and $E_{\max }$ are

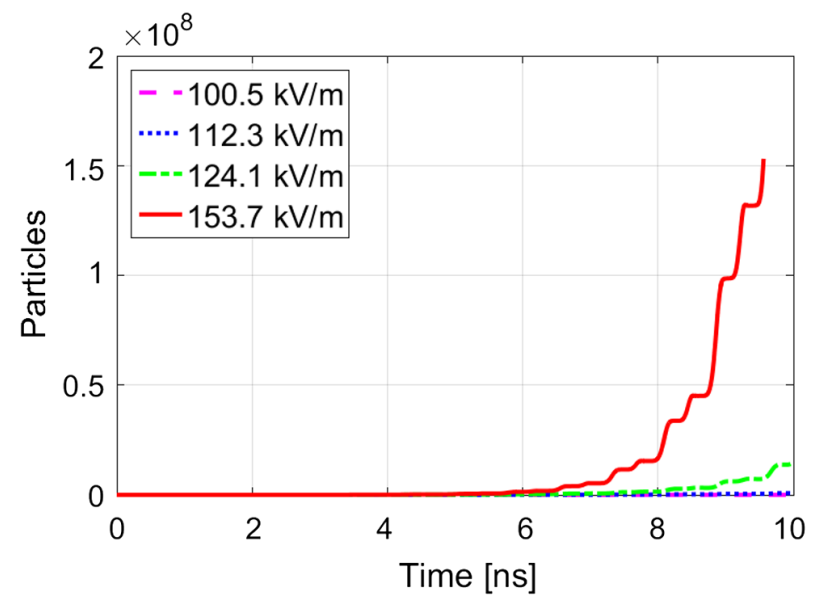

FIG. 16. Number of particles as a function of time (ns) for $1.5 \mathrm{~mm}$ gap width of the coaxial line structure.

$\sim 95$ and $\sim 336 \mathrm{kV} / \mathrm{m}$ with impact energies of $\sim 35$ and $206 \mathrm{eV}$, respectively. The large number of secondary electrons that were produced within the first $10 \mathrm{~ns}$ above 153.7 MV/m leads to a disproportionate computational time as shown in Fig. 16. Thus, it was quite reasonable to reduce the simulation time to $5 \mathrm{~ns}$ for each of the evaluations in order to calculate the $\mathrm{SEY}_{a}$ with respect to the lower and upper field limits as well as the corresponding impact energy. In comparison to the present gap of $0.75 \mathrm{~mm}$, the enlargement to $3 \mathrm{~mm}$ is considerably shifting MP upwards to higher field levels. The peak SEY $a$ ( 2.1) for example was generated at $353.8 \mathrm{kV} / \mathrm{m}$ with a corresponding impact energy of $387.3 \mathrm{eV}$. The same is true for $E_{\min }$ and $E_{\max }$ with $\sim 190$ and $\sim 596 \mathrm{kV} / \mathrm{m}$ as well as impact energies between $\sim 40.1$ and $712 \mathrm{eV}$. Very strong and consistent MP barriers were observed for 1.5 and $3 \mathrm{~mm}$ gaps. In addition, a larger gap shifts all fields to higher

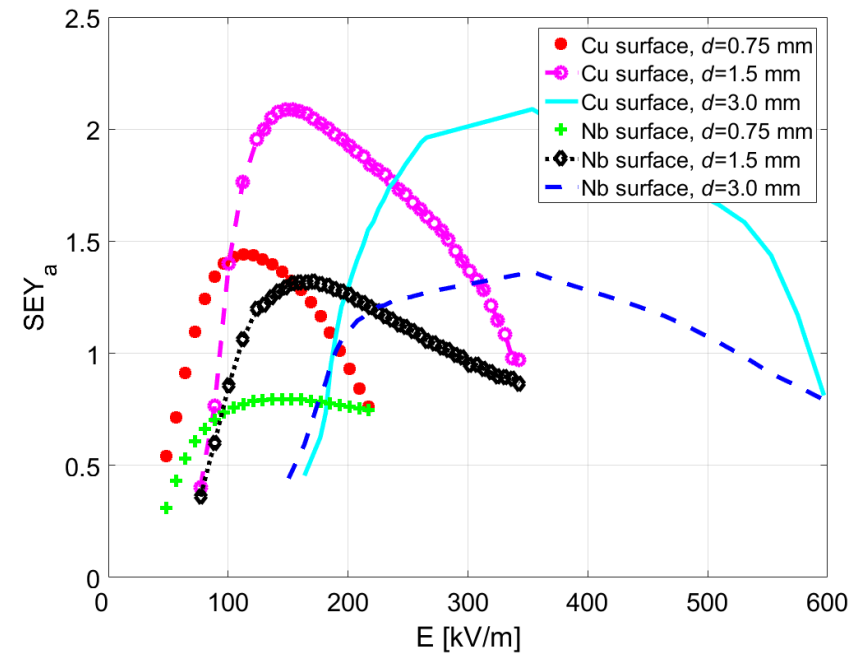

FIG. 17. Average SEYs of $\mathrm{Cu}$ and $\mathrm{Nb}$ surfaces as a function of the electric field amplitude for $0.75,1.5$, and $3 \mathrm{~mm}$ gap width of the coaxial line. 
values. However, the average peak SEYs are about the same for 1.5 and $3 \mathrm{~mm}$, although at different field level. In general, the field range in which MP occurs increased by a factor of approximately 1.8 when doubling the gap width. As Fig. 17 shows, a $3 \mathrm{~mm}$ gap improves the lower electric field threshold for MP comparing to the other two gap widths. However, MP will be even stronger and broader at higher fields. More specifically, as previously mentioned, for the latter gap, MP would occur between $E_{\min }$ and $E_{\max }$ with $\sim 190$ and $\sim 596 \mathrm{kV} / \mathrm{m}$ gap voltage corresponding to 2.66 to $8.2 \mathrm{MV} / \mathrm{m}$ in the half cell. These fields are still in the working range of the SRF gun. Therefore, due to the higher $\mathrm{SEY}_{a}$ and the broader field range where MP may occur, the bigger gaps are not preferable here.

\section{CONCLUSION}

The coaxial part of the photocathode channel of an SRF gun provides conditions conducive to MP due to the geometrical arrangement of the cathode region and the rf field leaking into it. We carried out numerical simulations in order to gain a better understanding of the multipactor phenomenon and to obtain MP suppression techniques for the SRF gun. Hitherto, a $\mathrm{Cu}$ photocathode and a $\mathrm{Nb}$ cavity combined with the advanced probabilistic model for the secondary emission process developed by Furman and Pivi have been employed. We used CST MWS and CST PS to compute the electromagnetic field and the electron multipactor discharge. A comparison between numerical simulations and analytical predictions done for a simplified plane-parallel model shows good agreement and indicates that two-sided first-order MP exists in the coaxial part of the cathode unit. The numerical study additionally revealed that for a $0.75 \mathrm{~mm}$ gap this type of MP occurs at low amplitudes typically between $\sim 70$ and $\sim 194 \mathrm{kV} / \mathrm{m}$. The highest generation of secondary electrons was observed at a gap field of $113 \mathrm{kV} / \mathrm{m}$ which corresponds to an accelerating field in the cavity of about $1.53 \mathrm{MV} / \mathrm{m}$. In particular, the latter confirmed that the simulation results are in good agreement with experimental data at ELBE.

Several possible remedies to suppress electron multipactor discharge and to improve the breakdown threshold in the vicinity of the photocathode of the SRF gun have been investigated. Introducing rectangular and triangular grooves on the cathode surface proved to be an efficient way to suppress vacuum electron MP. Both kinds of grooved surfaces ensure impact energies below the first crossover energy. This follows from reducing the $\mathrm{SEY}_{a}$ below the multipactor threshold. We presented simulation results with an optimized cathode model. With the optimal parameters for the rectangularly grooved surface, the $\delta_{\max }$ for $\mathrm{Cu}$ was reduced from 1.44 to 0.956 while the isosceles triangularly grooved surface reduces the SEY from 1.44 to 0.91 . These were both measured at $113 \mathrm{kV} / \mathrm{m}$ where the highest MP was observed for a flat surface. Thus, both types of grooved surfaces are effective in reducing the SEY below the MP threshold and thus suppressing MP in the cathode region.

DC biasing and a geometry modification are the other attempted multipactor suppression methods. The DC biasing voltage was applied according to the scaling law based on our own previous studies. The results showed that MP could be suppressed employing very low voltages. Typically, $100 \mathrm{~V}$ achieve MP suppression after $1.5 \mathrm{~ns}$. However, these results do not match the experimental data gained at ELBE. Thus, further numerical and experimental studies might be required in order to investigate this discrepancy between numerical and experimental results. Moreover, we investigated a variation of the dimension of the coaxial part of the structure in order to study MP behavior and field thresholds to it. A practical aim of this approach is to provide more space in the photocathode unit to allow for a better cleaning process. Varying the diameter of the outer conductor showed that MP bands shift to higher field levels with larger gap widths. Although a larger gap improves the breakdown threshold at lower fields, strong MP occurs at a higher field level.

\section{ACKNOWLEDGMENTS}

The authors would like to thank Dr. Erion Gjonaj, Prof. Jens Knoblauch and the other members of the HOPE consortium for valuable discussions and comments. This work was supported by the Federal Ministry for Research and Education BMBF under Project No. 05K2013-HOPE.

\section{APPENDIX A: ANALYTICAL MODEL FOR TWO-SIDED MULTIPACTOR}

A simple analytical model for two-sided multipactor was set up for the coaxial part of the structure, where MP is critical as denoted above. For local inspection of a small volume, two parallel plates can approximate the coaxial line structure to predict the order of MP and the range of the electric field in which MP may occur. Both the magnetic field and the space charge effects can be ignored since their effects are negligible here $[13,11]$.

The resonant condition for two-sided MP requires that the transit time of the electron to the outer conductor is an odd integer number of the half rf period [13]

$$
t=\frac{(2 n-1) \pi}{\omega_{g}}=\frac{T}{2}(2 n-1), \quad n=1,2,3, \ldots
$$

where $T$ is the rf period and $n$ is the order of MP. The voltage in the gap of the two parallel plates $\left(V_{g}\right)$ in case of two-sided MP can be calculated by

$$
V_{g}=E_{0} d=\frac{4 \pi m_{e} c^{2} d^{2}}{(2 n-1) e \lambda^{2}}
$$


TABLE I. Analytical and numerical solution for the first order two-sided MP.

\begin{tabular}{|c|c|c|c|c|c|}
\hline & \multicolumn{3}{|c|}{ Numerical Solution } & \multicolumn{2}{|c|}{ Analytical Solution } \\
\hline & $\mathrm{E}[\mathrm{kV} / \mathrm{m}]\left(\right.$ at $\mathrm{SEY}_{a}$ peak) & $\mathrm{U}[\mathrm{eV}]$ (at $\mathrm{SEY}_{a}$ peak) & $\mathrm{SEY}_{a}$ (peak) & $\mathrm{E}[\mathrm{kV} / \mathrm{m}]$ & $\mathrm{U}[\mathrm{eV}]$ \\
\hline \multicolumn{6}{|l|}{ Flat surface } \\
\hline $0.75 \mathrm{~mm}$ & 112.7 & 45.6 & 1.440 & 90.3 & 43.1 \\
\hline $1.5 \mathrm{~mm}$ & 153.7 & 387.3 & 2.100 & 180.5 & 172.4 \\
\hline $3 \mathrm{~mm}$ & 353.8 & 387.3 & 2.100 & 361.0 & 689.5 \\
\hline \multicolumn{6}{|l|}{ Rectangular surface } \\
\hline $0.75 \mathrm{~mm}$ & 112.9 & 22.6 & 0.956 & $\cdots$ & $\cdots$ \\
\hline \multicolumn{6}{|l|}{ Isosceles surface } \\
\hline $0.75 \mathrm{~mm}$ & 113.0 & 15.6 & 0.910 & $\cdots$ & $\cdots$ \\
\hline
\end{tabular}

and the corresponding impact energy $(U)$ is given by

$$
U=\frac{8 m_{e} c^{2} d^{2}}{(2 n-1)^{2} \lambda^{2}},
$$

where $c$ is the speed of light and $\lambda$ is the rf wave length. Thus, by using the last two equations, the prediction of a possible two-sided MP of $n$th order is possible in the vicinity of the cathode.

Analytical and numerical results for the flat surface, and the rectangularly and the isosceles triangularly grooved surfaces are summarized in Table I. Simulation results are recorded for the gap widths $d$ of $0.75 \mathrm{~mm}, 1.5 \mathrm{~mm}$ and $3 \mathrm{~mm}$ in case of a flat surface and for the gap width $d$ of $0.75 \mathrm{~mm}$ in case of rectangularly and the isosceles triangularly grooved surfaces for $\mathrm{Cu}$ material at the operating frequency of $1.3 \mathrm{GHz}$. A larger gap improves the threshold voltage and electric field.

\section{APPENDIX B: TRUST-REGION ALGORITHM}

The trust-region algorithm works as follows: Step 0. Choose initial point $\left(x_{0}\right)$ and set an initial trust-region radius $\Delta_{k}$ for the $k^{\text {th }}$ iteration (initially $k=1$ ).

Step 1. Calculate the trial step $\left(s_{k}\right)$ by solving the trustregion subproblem

$\min _{s \in \Re^{n}} m_{k}(s)$ subject to $\|s\| \leq \Delta_{k}$ to reduce $f\left(x_{k}+s\right)$.

$\|s\|$ is the trust-region constraint, where $\|$.$\| is the standard$ Euclidean norm.

Step 2. Decide whether to set $x_{k+1}=x_{k}+s_{k}$ or $x_{k+1}=$ $x_{k}$ based on the reduction of the goal function.

Step 3. Construct a new model $m_{k+1}(s)$ for the next iteration, choose $\Delta_{k+1}$ and set $k$ to $k+1$ and go to step 1 .

[1] R. A. Kishek, Y. Y. Lau, L. K. Ang, A. Valfells, and R. M. Gilgenbach, Multipactor discharge on metals and dielectrics: Historical review and recent theories, Phys. Plasmas 5, 2120 (1998).
[2] C. Chang, G. Liu, C. Tang, C. Chen, and J. Fang, Review of recent theories and experiments for improving highpower microwave window breakdown thresholds, Phys. Plasmas 18, 055702 (2011).

[3] C. Gutton, Sur la décharge électrique à fréquence très élevée, em Comptes-Rendus Hebdomadaires des Séances de l'Académie des 178, 467 (1924).

[4] P. Farnsworth, Television by electron image scanning, J. Franklin Inst. 218, 411 (1934).

[5] E. W. B. Gill and A. von Engel, Starting potentials of highfrequency gas discharges at low pressure, Proc. R. Soc. Lond. A 192, 446 (1948)

[6] A. J. Hatch and H. B. Williams, The secondary electron resonance mechanism of low-pressure high-frequency gas breakdown, J. Appl. Phys. 25, 417 (1954).

[7] A. J. Hatch and H. B. Williams, Multipacting modes of highfrequency gaseous breakdown, Phys. Rev. 112, 681 (1958).

[8] V.D. Shemelin, Existence zones for multipactor discharges, Sov. Phys. Tech. Phys. 31, 1730 (1986).

[9] J. R. M. Vaughan, Multipactor, IEEE Trans. Electron Devices 35, 1172 (1988).

[10] V. Shemelin, Generalized phase stability in multipacting, Phys. Rev. ST Accel. Beams 14, 092002 (2011).

[11] G. Torregrosa, Á. Coves, C. P. Vicente, A. M. Pérez, B. Gimeno, and V. E. Boria, Time evolution of an electron discharge in a parallel-plate dielectric-loaded waveguide, IEEE Electron Device Lett. 27, 619 (2006).

[12] M. A. Furman and M. T. F. Pivi, Probabilistic model for the simulation of secondary electron emission, Phys. Rev. ST Accel. Beams 5, 124404 (2002).

[13] H. Padamsee, J. Knobloch, and T. Hays, RF Superconductivity for Accelerators, 2nd ed. (John Wiley and Sons, New York, 2011).

[14] V. Shemelin, Conditions for the existence of 1-and 2-point multipactor in SRF cavities, Proceedings, 4th International Particle Accelerator Conference (IPAC 2013), Shanghai, China, 2013 (JACoW, Geneva, Switzerland, 2013), WEPWO067, http://JACoW.org/IPAC2013/papers/ wepwo067.pdf.

[15] H. C. Kim and J. P. Verboncoeur, Transition of window breakdown from vacuum multipactor discharge to $\mathrm{rf}$ plasma, Phys. Plasmas 13, 123506 (2006).

[16] P. Zhang, Y. Y. Lau, M. Franzi, and R. M. Gilgenbach, Multipactor susceptibility on a dielectric with a bias 
dc electric field and a background gas, Phys. Plasmas 18, 053508 (2011).

[17] A. Iqbal, J. Verboncoeur, and P. Zhang, Multipactor susceptibility on a dielectric with two carrier frequencies, Phys. Plasmas 25, 043501 (2018).

[18] A. Arnold, H. Buttig, D. Janssen, T. Kamps, G. Klemz, W. D. Lehmann, U. Lehnert, D. Lipka, F. Marhauser, P. Michel, K. Moller, P. Murcek, Ch. Schneider, R. Schurig, F. Staufenbiel, J. Stephan, J. Teichert, V. Volkov, I. Will, and R. Xiang, Development of a superconducting radio frequency photoelectron injector, Nucl. Instrum. Methods Phys. Res. Sect. A 577, 440 (2007).

[19] A. Arnold and J. Teichert, Overview on superconducting photoinjectors, Phys. Rev. ST Accel. Beams 14, 024801 (2011).

[20] J. Teichert, A. Arnold, H. Büttig, U. Lehnert, P. Michel, P. Murcek, Ch. Schneider, R. Schurig, G. Staats, R. Xiang, P. Kneisel, T. Kamps, J. Rudolph, M. Schenk, G. Klemz, and I. Will, Operation of the superconducting if photo gun at ELBE, J. Phys. Conf. Ser. 298, 012008 (2011).

[21] CST-Computer Simulation Technology AG. CST STUDIO SUITE 2017. Bad Nauheimer Str. 19, 64289 Darmstadt, Germany. https://www.cst.com.

[22] P. Berutti, T. Khabiboulline, and G. Romanov, Multipactor Discharge in the PIP-II Superconducting Spoke Resonators, Fermilab, Technical division, Technical note TD-16005, 2016.

[23] G. Romanov, P. Berrutti, and T. N. Khabiboulline, Simulation of multipacting in SC low beta cavities at FNAL, Proceedings, 6th International Particle Accelerator Conference (IPAC 2015), Richmond, Virginia, USA, 2015 (JACoW, Geneva, Switzerland, 2015), MOPMA018, DOI: 10.18429/JACoW-IPAC2015-MOPMA018.

[24] G. Romanov, Update on multipactor in coaxial waveguides using CST Particle Studio, in Proceedings of the 24th Particle Accelerator Conference, PAC-2011, New York, 2011 (IEEE, New York, 2011).

[25] U. Van Rienen, Numerical Methods in Computational Electrodynamics: Linear Systems in Practical Applications (Springer Science \& Business Media, New York, 2012), Vol. 12.

[26] A. di Bona, F. Sabary, S. Valeri, P. Michelato, D. Sertore, and G. Suberlucq, Auger and x-ray photoemission spectroscopy study on Cs2Te photocathodes, J. Appl. Phys. 80, 3024 (1996).

[27] P. Michelato, Photocathodes for RF Photoinjectors, Nucl. Instrum. Methods Phys. Res., Sect. A 393, 455 (1997).

[28] A. Arnold (HZDR) (private communication).

[29] W. Xu, Z. Altinbas, S. Belomestnykh, I. Ben-Zvi, M. Cole, S. Deonarine, M. Falletta, J. Jamilkowski, D. Gassner, P. Kankiya et al., Design, simulations, and conditioning of $500 \mathrm{kw}$ fundamental power couplers for a superconducting rf gun, Phys. Rev. ST Accel. Beams 15, 072001 (2012).

[30] S. Wang, Secondary electron yield measurements of anti-multipacting surfaces for accelerators, Ph.D. thesis, Loughborough University, 2016.

[31] R. Valizadeh, O. B. Malyshev, S. Wang, S. A. Zolotovskaya, W. Allan Gillespie, and A. Abdolvand, Low secondary electron yield engineered surface for electron cloud mitigation, Appl. Phys. Lett. 105, 231605 (2014).
[32] E. Hoyt, M. Hoyt, R. Kirby, C. Perkins, D. Wright, and A. Farvid, Processing of OFE copper beam chambers for PEP-II high energy ring, in Proceedings of the Particle Accelerator Conference, Dallas, TX, 1995 (IEEE, New York, 1995), Vol. 3, pp. 2054-2056.

[33] U. Klein and D. Proch, Multipactoring in superconducting rf structures, in Proceedings, Conference on Future Possibilities for Electron Accelerators, Charlottesville, USA, 1979 (1979), Vol. N1-17.

[34] J. Knobloch, W. Hartung, and H. Padamsee, Multipacting in $1.5-\mathrm{GHz}$ superconducting niobium cavities of the CEBAF shape, in $R F$ superconductivity. Proceedings, 8th Workshop, Abano Terme, Padua, Italy, 1997. Vol. 1: Superconducting particle accelerators. Vol. 2: Surface resistance and non-accelerator applications. Vol. 3: Resonator construction technologies. Vol. 4: Superconducting materials and thin films (1997), pp. 1017-1027, http:// www.lns.cornell.edu/public/SRF/1998/SRF981012-09.

[35] P. Yla-Oijala and M. Ukkola, Suppressing electron multipacting in ceramic windows by DC bias, Nucl. Instrum. Methods Phys. Res., Sect. A 474, 197 (2001).

[36] R. L. Geng, H. Padamsee, S. Belomestnykh, P. Goudket, D. M. Dykes, and R. G. Carter, Suppression of multipacting in rectangular coupler waveguides, Nucl. Instrum. Methods Phys. Res., Sect. A 508, 227 (2003).

[37] D. Gonzalez-Iglesias, A. M. Perez, S. Anza, J. Vague, B. Gimeno, V. E. Boria, D. Raboso, C. Vicente, J. Gil, F. Caspers, et al.Multipactor in a coaxial line under the presence of an axial dc magnetic field, IEEE Electron Device Lett., 33, 727 (2012).

[38] D. González-Iglesias, A. M. Pérez, S. Anza, J. V. Cardona, B. Gimeno, V. E. Boria, D. Raboso, C. Vicente, J. Gil, F. Caspers et al., Multipactor mitigation in coaxial lines by means of permanent magnets, IEEE Trans. Electron Devices 61, 4224 (2014).

[39] L. Wang, T. O. Raubenheimer, and G. Stupakov, Suppression of secondary emission in a magnetic field using triangular and rectangular surfaces, Nucl. Instrum. Methods Phys. Res., Sect. A 571, 588 (2007).

[40] M. Pivi, F. K. King, R. E. Kirby, T. O. Raubenheimer, G. Stupakov, and F. Le Pimpec, Sharp reduction of the secondary electron emission yield from grooved surfaces, J. Appl. Phys. 104, 104904 (2008).

[41] G. Stupakov and M. Pivi, Supperssion of the Effective Secondary Emission Yield for a Grooved Metal Surface, CERN Reports No. LCC-0145, No. SLAC-TN-04-045, 2004.

[42] A. A. Krasnov, Molecular pumping properties of the LHC arc beam pipe and effective secondary electron emission from $\mathrm{Cu}$ surface with artificial roughness, Vacuum 73, 195 (2004); Proceedings of the European Vacuum Congress Berlin 2003, featuring the 8th European Vacuum Conference, 2nd Annual Conference of the German Vacuum Society.

[43] T. Abe, T. Kageyama, H. Sakai, Y. Takeuchi, and K. Yoshino, Multipactoring suppression by fine grooving of conductor surfaces of coaxial-line input couplers for high beam current storage rings, Phys. Rev. ST Accel. Beams 13, 102001 (2010).

[44] W. Xu, S. Belomestnykh, I. Ben-Zvi, C. Liaw, K. Smith, R. Than, J. Tuozzolo, E. Wang, D. Weiss, and A. Zaltsman, 
Multipacting-free quarter-wavelength choke joint design for BNL SRF, in Proceedings, 6th International Particle Accelerator Conference (IPAC 2015): Richmond, Virginia, USA, 2015 (JACoW, Geneva, Switzerland, 2015), TUPMA047, DOI: 10.18429/JACoW-IPAC2015TUPMA047.

[45] E. T. Tulu, U. van Rienen, and A. Arnold, Different countermeasures of electron amplification in the photocathode unit, in Proceedings of 5th International Particle Accelerator Conference (IPAC 2014): Dresden, Germany, 2014 (JACoW, Geneva, Switzerland, 2014), MOPRI028 http://jacow.org/IPAC2014/papers/mopri028.pdf.

[46] E. Somersalo, P. Yla-Oijala, D. Proche, and J. Sarvas, Computational methods for analyzing electron multipacting in rf structures, Part. Accel. 59, 107 (1998).
[47] E. T. Tulu, A. Arnold, and U. van Rienen, Numerical studies on a modified cathode tip for the ELBE superconducting rf gun, in Proceedings of International Particle Accelerator Conference (IPAC2017), Copenhagen, Denmark, 2017 (JACOW, Geneva, Switzerland, 2007), pp. 515-517, http://accelconf.web.cern.ch/AccelConf/ipac2017/papers/ mopik008.pdf.

[48] A. R. Conn, N. I. M. Gould, and P. L. Toint, Trust-Region Methods (SIAM, 2000), Vol. 1.

[49] N. I. M. Gould and S. Leyffer, An Introduction to Algorithms for Nonlinear Optimization (Springer, New York, 2003).

[50] Y. Yuan, A review of trust region algorithms for optimization, in Iciam (Oxford University Press, Oxford, New York, 2000), Vol. 99, pp. 271-282. 